\title{
ILCEA
}

Revue de l'Institut des langues et cultures

d'Europe, Amérique, Afrique, Asie et Australie

$20 \mid 2014$

Le conte : d'un art à l'autre

\section{Les contes vus par le cinéma soviétique (années 1930-1950)}

Tales Seen by the Soviet Cinema (1930-1950s)

СКАЗКИ В СОВЕТСКОМ КИНЕМАТОГРАФЕ 1930-1950-х rr

\section{Galina Kabakova}

\section{OpenEdition}

Journals

Édition électronique

URL : http://journals.openedition.org/ilcea/2725

DOI : 10.4000/ilcea.2725

ISSN : 2101-0609

Éditeur

UGA Éditions/Université Grenoble Alpes

Édition imprimée

ISBN : 978-2-84310-290-5

ISSN : 1639-6073

Référence électronique

Galina Kabakova, «Les contes vus par le cinéma soviétique (années 1930-1950) », ILCEA [En ligne],

20 | 2014, mis en ligne le 08 décembre 2014, consulté le 19 avril 2019. URL : http://

journals.openedition.org/ilcea/2725; DOI : 10.4000/ilcea.2725

Ce document a été généré automatiquement le 19 avril 2019

(C) ILCEA 


\title{
Les contes vus par le cinéma soviétique (années 1930-1950)
}

\author{
Tales Seen by the Soviet Cinema (1930-1950s) \\ СКАЗКИ В СОВЕТСКОМ КИНЕМАТОГРАФЕ 1930-1950-х rr
}

Galina Kabakova

1 L'industrie du cinéma soviétique conçu dès le départ comme un outil de propagande et d'éducation des masses, a produit le tout premier film pour enfants en 1918. Le film Signal , perdu comme la majeure partie des films muets, devait expliquer aux enfants le sens du sacrifice au nom de la révolution. Des films pour la jeunesse apparaissent ponctuellement, mais la vraie production à destination des enfants commence au milieu des années 1920. Les cinéastes portent à l'écran les aventures de pionniers qui rendent visite à Lénine ou de jeunes héros de la guerre civile et le western révolutionnaire les Diablotins rouges (1923) devient vite un blockbuster. Mais à cette époque, le conte traditionnel suscite la méfiance des autorités car à leurs yeux, d'une part, il ne véhicule pas les valeurs de la nouvelle société et d'autre part, le merveilleux est jugé nocif pour la conscience des jeunes bâtisseurs du socialisme. Ainsi, en 1924, Nadejda Kroupskaïa, la compagne de Lénine et présidente de Glavpolitprosvet, la délégation du ministère de l'Instruction publique en charge de l'éducation politique, interdit la publication et même la conservation dans les bibliothèques des livres de contes, s'appuyant sur l'avis de psychologues radicaux, auteurs des études aux titres parlants «Nous sommes contre le conte» ou encore «L'enfant prolétaire a-t-il besoin du conte ?» (E. Yanovskaïa) (Kabakova, 2005 : 143-148).

2 La réhabilitation du conte - et du folklore en général - ne se fera que dix ans plus tard dans le contexte du retour aux valeurs conservatrices. Comme l'écrit Katerina Clark, le culte du positivisme est remplacé par le culte de l'idéologie officielle en tant que dogme quasi religieux, mystique, supérieur au rationnel (Clark, 1985: 141). En 1934, au $\mathrm{I}^{\mathrm{er}}$ congrès d'écrivains soviétiques, le conte recouvre ses lettres de noblesse et sera désigné comme le genre principal de la littérature jeunesse. En 1936, le président du Commissariat aux arts (ministère de la Culture) Platon Kerjentsev va pester contre les éditeurs qui tardent à rééditer les contes d'Afanassiev et d'autres recueils du folklore. Le 
folklore sera dorénavant utilisé comme un moyen d'éducation autant que de divertissement. Après l'interdiction de l'opéra-comique de Demian Bedny Les Preux en 1936, se moquant des héros des bylines (chants épiques), personne n'osera tourner en dérision les personnages héroïques du folklore russe au risque de se voir exclu du Parti communiste comme Bedny.

3 Car dorénavant le folklore en général, le conte et les bylines en particulier, joueront un rôle non négligeable dans la promotion du patriotisme soviétique aux accents de plus en plus nationalistes. À partir de 1938, les héros de la littérature orale d'avant la Révolution envahissent les écrans; à la pointe de la lutte idéologique, ils sont appelés à recréer l'image du passé glorieux de la Russie et à intimider les ennemis potentiels. Ne citons qu' Alexandre Nevski de Serguei Eisentsein (1938), Sadko (1952), et Ilya Mouromets de Ptouchko (1956). En effet, dans Alexandre Nevski, qui n'est pas destiné aux enfants, on trouve le personnage historique, épaulé par le preux emprunté aux bylines, Vassili Bouslaï mais aussi, fait marquant, une référence au conte licencieux « Le renard et le lièvre » du recueil Contes secrets russes d'Alexandre Afanassiev publié à l'étranger en 1872 pour des raisons de censure. Ce petit conte scabreux, racontant la mésaventure d'une renarde abusée par le lièvre et récité par un personnage du film, suggère au prince Alexandre Nevski le moyen de piéger les chevaliers teutoniques et d'en venir à bout.

Ce tournant idéologique se concrétise à travers la réalisation de plusieurs ciné-contes par les très talentueux Alexandre Ro'ou (1906-1973) et Alexandre Ptouchko (1900-1973), inventeurs de ce genre en URSS. Le premier en date est Par l'ordre du brochet de Ro'ou (1938) qui représente la contamination de trois contes populaires russes. La notion de ciné-conte englobait aussi bien les contes populaires russes, que des contes devenus populaires grâce aux traductions ou adaptations, tels Cendrillon, La Reine des neiges ou La Fille de Neige, conte international adapté par Alexandre Ostrovski, ou encore des contes littéraires comme Le Petit cheval bossu, libre adaptation par Petr Erchov des contes russes et nordiques, ou encore La Fleur de pierre de Pavel Bajov, inspiré du folklore de l'Oural. Dans cette catégorie, sont classées également des œuvres de littérature pour la jeunesse, comme La Petite clé d'or, adaptation du Pinocchio de Carlo Collodi par Alexeï Tolstoï, mais également des scénarios originaux qui brodent avec plus au moins d'habileté sur la trame du conte merveilleux.

5 La guerre permet de réaliser pleinement le potentiel idéologique du conte et de l'épopée, car ils servent de prétexte afin de mobiliser le peuple soviétique pour lutter contre le nazisme. Le film d'Alexandre Ro'ou Kachtcheï l'Immortel (1944) présente l'exemple le plus saillant de l'usage politique du folklore au cinéma. Par ailleurs, la première du film a lieu le 9 mai 1945, le jour de la Victoire, à Barnaoul. Dans le film, le monstre Kachtcheï ne se contente pas de captiver la belle Maria Morevna, il met le feu à la terre russe. Et le conte présente dès le début les accents belliqueux d'une byline. Le rôle de libérateur du pays est confié à Nikita Kojemiaka, variante folklorique de saint Georges. La leçon de patriotisme est inculquée par tous les moyens: des mains d'un champignon, le héros reçoit une poignée de sa terre natale en tant que don merveilleux qui le rend invincible. En guise d'épreuve préalable, il doit résoudre l'énigme suivante : qui doit-on aimer plus que ses père et mère ? Réponse : la patrie, etc.

Ce patriotisme aux accents russes permet de procéder à la réhabilitation du folklore russe dans son ensemble, et surtout à la réhabilitation de la religion, comme dans la scène où les enfants qui chantent les chansons célébrant le début du printemps : ce n'est pas par hasard que l'aède au début du film chante la gloire de la Sainte Russie. Le film n'hésite pas 
à mettre en scène la double foi : on voit la petite chapelle incendiée par les chevaliers teutoniques et, à côté, des idoles païennes en bois également saccagées par l'ennemi, sont montrées avec autant de sympathie que la chapelle, symbole de la religion nationale.

7 Sur le plan esthétique, Ro'ou s'inspire largement du cinéma héroïque d'Eisenstein et avant tout d'Alexandre Nevski. En même temps, en dénonçant le nazisme, il emprunte beaucoup à l'expressionisme allemand, ce qui fait du film, aux yeux de certains historiens du cinéma, le premier film d'horreur, genre en principe absent du cinéma soviétique. Le château de Kachtcheï semble sortir d'un film de Murnau ou de Lang, et selon l'aveu de Grigorij Milliar, l'interprète attitré de tous les méchants du conte merveilleux, le réalisateur a pris pour cible les Nibelungen de Fritz Lang. En effet, dans le maquillage de Kachtcheï, on peut reconnaitre Alberich, le roi des Nibelungen, tout comme l'un des cavaliers de l'Apocalypse, sorti directement du tableau éponyme de Victor Vasnetsov. Par ailleurs, le cinéma de Lang avait déjà inspiré le cinéaste dans sa réalisation précédente Vassilissa la Belle où Ivachka, tel Siegfried, combat vaillamment le dragon. D'ailleurs, Ro'ou n'est pas le seul à appliquer les recettes esthétiques de l'expressionisme ; on les retrouve également dans d'autres films des années 1930-1940 avant que le cinéma soviétique n'élabore sa propre esthétique du merveilleux.

conte cinématographique ne fait pas que dévoiler les ennemis extérieurs, il fait également écho aux grands sujets de société et dénonce les ennemis intérieurs. Ainsi, dans la Graine magique (1941), on retrouve le thème du pays de cocagne identifié à l'URSS. Ici c'est le paradis terrestre qui sort d'une graine magique offerte par un preux aux enfants. En effet, en faisant pousser la betterave géante qui produira tout le sucre nécessaire pour le pays, symbole de la richesse, les enfants doivent affronter les méchants charançons qui sont la parfaite incarnation des saboteurs de tout acabit. Ils s'approchent de la plante magique tels les trolls de Peer Gynt et causent des dégâts considérables avant d'être démasqués par les enfants vigilants. Le beau preux ordonne au saboteur de dévoiler sa vraie nature (« sois ce que tu es en réalité » d'après la célèbre formule), ce qui cause sa perte. Il faut savoir que la lutte contre la vermine était à l'ordre du jour aussi bien au sens figuré, sur le plan politique, qu'au sens propre.

Parmi les modalités d'adaptation du conte, la tendance principale était la politisation très forte de tous les genres y compris le ciné-conte. La propagande politique était menée tambour battant: par exemple, dans l'adaptation de La Petite clé d'or (1939), un film de Ptouchko, d'après le scénario d'Alexeï Tolstoï, la clef magique ouvrait la porte cachée qui donnait non plus sur un théâtre de marionnettes mais sur un livre merveilleux. Sa page de titre représentait le Kremlin: l'avenir radieux s'ouvrait devant les personnages opprimés par le système capitaliste et qui avaient la chance inouie d'être transportés par la magie dans ce royaume de justice sociale. Dans une adaptation ou plutôt réécriture du chef-d'œuvre de Swift, Le Nouveau Gulliver de Ptouchko (1935), on découvre une révolution prolétarienne qui couve dans le pays des Lilliput.

10 Et lorsque la période stalinienne prend fin, pendant la période du Dégel, les réalisateurs profitent également du conte pour envoyer des piques : ainsi dans L'Habile Maria (1960) l'héroïne éponyme est anesthésiée pour longtemps par la formule magique «Liberté ou servilité c'est du pareil au même ». Evguenij Schwartz, le scénariste, et Alexandre Ro'ou, le réalisateur, faisaient bien évidemment référence au pays tout entier transformé en zombi par le régime stalinien.

De même, on attendait des réalisateurs que leurs créations inculquent les valeurs morales et patriotiques de bâtisseurs de la société socialiste, selon la formule célèbre d'Alexandre 
Pouchkine «Tout conte est mensonge mais n'en contient pas moins quelque allusion. Puisse-t-il servir de leçon à maints braves jeunes gens. ». Ainsi, on incruste dans la trame narrative des leçons de morale. Ne citons qu'un exemple: dans Le Père Frimas (1964) de Ro'ou, le héros Ivanouchka, en punition de sa vantardise, se fait transformer en ours par un sorcier et le sort ne sera levé que quand il aura accompli trois bonnes actions.

Une autre règle respectée par les cinéastes est la pureté sociologique des personnages. Déjà à l'aube de l'État soviétique, on reprochait au conte l'omniprésence des tsars et des princes. Une fois le conte réhabilité, la politique des classes est rigoureusement appliquée aux personnages : tsars et tsarines peuvent être ridicules dans le meilleur des cas, mais surtout bêtes et méchants. Les princes subissent la purge la plus dure: comme dans le système des personnages du conte, le prince ne peut être que le héros, et que dans le système du politiquement correct à la socialiste, le héros ne peut appartenir qu'aux classes populaires, tous les princes des contes de fées se voient rétrogradés en hommes du peuple : ainsi, dans Vassilissa la Belle d'Alexandre Ro'ou (1939), adaptée du conte Princesse Grenouille, le prince Ivan devient simplement Ivan et en cours de route, il se métamorphose en preux ; la Grenouille enchantée devient une belle paysanne et non plus une princesse. Il faut quand même préciser que les princesses sont mieux loties que les princes car elles peuvent être rééduquées par l'amour de leurs roturiers de fiancés. Les représentants des classes autrefois dominantes, nobles ou marchands, apparaissent également comme des personnages satiriques, à l'exception peut-être du Cendrillon de Nadejda Kocheverova et Mikhaïl Chapiro (1947).

Le troisième trait qui caractérise ce cinéma de contes de fées, c'est la cyclisation très forte et amorcée dès les premiers films. En effet, l'industrie du film merveilleux connaît son âge d'or grâce aux créations d'Alexandre Ptouchko et d'Alexandre Ro'ou, les vrais pionniers du cinéma pour enfants. Or, ils ont imposé leurs comédiens qui jouaient le même type de rôles d'un film à l'autre. Ainsi, l'un des comédiens fétiches d'Alexandre Ro'ou, Grigorij Milliar (fils d'un ingénieur français, François de Milieu), s'est produit dans tous les rôles de méchants, de Kachtcheï à Baba Yaga, et dans plusieurs rôles de vieillards. Souvent, le réalisateur lui confiait jusqu'à trois rôles dans un même film, comme dans Le Petit cheval bossu (1941), où il interprète conteur, brigand, et chambellan.

De même, en face d'un vrai méchant, on trouve un vrai preux aryen, interprété par le comédien Serguei Stoliarov (Rouslan dans Rouslan et Liudmila ; Ivan dans Vassilissa la Belle; Nikita Kojemiaka dans Kachtcheï l'Immortel; Sadko dans le film éponyme; Aliocha Popovitch dans Ilya Mouromets). Par ailleurs, ce comédien moyennement doué, mais qui incarnait le mieux l'idéal de la virilité, a servi de modèle à Vera Moukhina pour sa célèbre statue L'ouvrier et la kolkhozienne.

Enfin, certains éléments de la structure des films produisent également cet effet de cyclisation. Par exemple, l'introduction de personnages inédits dans la poétique du conte qui passent sans encombre d'un film à l'autre : comme l'ours qui soumet le héros à une épreuve de force (Sadko, Vassilissa la Belle, entre autres) ou qui devient son auxiliaire ( L'Habile Maria), ou le champignon-donateur (Kachtcheï, Le Père Frimas), ou bien encore, les pirates qui peuplent allègrement plusieurs contes de Ro'ou (Barbara la fée aux cheveux de soie, L'Habile Maria).

La transposition de contes traditionnels, tout comme la création de scénarios originaux, obéissent à deux grands principes. Les contes ont tendance à épouser le type de contes privilégié par Vladimir Propp dans ses études: le récit construit autour du héros 
masculin. Ainsi, les contes typiquement "féminins", qui font partie du conte-type (A.T.U. 480), Vassilissa la Belle ou Le Père Frimas (Morozko), sont-ils "renforcés » par un autre conte type, cette fois-ci avec un homme dans le rôle principal. Il est légitime de s'interroger sur cette particularité de la réécriture. Il y a tout lieu de penser que l'explication ne réside pas dans l'autorité de Propp, ignoré à l'époque, mais dans la poétique du réalisme socialiste avec son culte de la Grande famille: au sein de cette famille, le rôle central est toujours attribué au héros et au père. Notons que Cendrillon a échappé à cette réécriture « masculinisante ».

Une autre spécificité concerne la place des enfants dans le récit cinématographique. Comme le cahier des charges consistait à créer le genre du cinéma pour enfants, les réalisateurs l'ont fait en introduisant dans les récits des protagonistes enfants, héros plutôt rares dans les contes populaires. L'exemple typique représente L'Habile Maria où un garçon est à la recherche de sa mère prisonnière dans le royaume sous-marin. On découvre le même procédé dans une des premières œuvres de Ptouchko, Le Nouveau Gulliver (1935), un film d'animation, où le réalisateur introduit dans la narration le pionnier Petia qui s'assoupit à la lecture des Voyages de Gulliver et se réveille au milieu de l'action.

La réécriture ne visait pas qu'un conte-type particulier mais l'ensemble des contes. Plus le temps passait, plus les contes devenaient des mosaïques résultant de l'assemblage de plusieurs contes. Vassilissa la Belle est un mélange de La Princesse Grenouille et de Vassilissa la Sage; Le Père Frimas est constitué du Père Frimas et de La Belle et la bête. D'autres films sont carrément des cross-over où l'on reconnait encore des personnages et des motifs du folklore russe mais pas les contes d'origine (Feu, eau et tuyaux de cuivre).

Les réalisateurs ont créé leur propre poétique du conte cinématographique, très influencée par le cinéma en vogue à l'époque. Ainsi la musique est omniprésente dans leurs œuvres comme dans tous les films de l'époque. Elle sert de pendant aux formules d'introduction et de clôture : les aèdes munis de leurs cithares donnent le la, et les autres personnages y compris les méchants prennent le relais en chantant ${ }^{1}$ et en dansant. Certains contes comme Rouslan et Lioudmila ressemblent à un opéra filmé. Ces réjouissances musicales participaient activement à la mise en scène du bonheur absolu. Rappelons que la phrase «La vie est devenue meilleure, camarades, la vie est devenue plus joyeuse », prononcée par Staline en janvier 1935, a défini pour longtemps la politique générale dans tous les domaines.

20 L'autre spécificité tient à la place accordée au texte, qui peut être extrêmement importante, ce qui est tout aussi typique de la culture stalinienne qui propulse au sommet des arts l'art poétique. Les textes rimés composés d'expressions populaires ou stylisées envahissent les écrans. Le cinéma pour enfants n'est pas que parlant, il est bavard.

Ptouchko et Ro'ou avaient à leur disposition une vraie industrie cinématographique et comme l'éducation des jeunes a toujours figuré parmi les priorités idéologiques, les studios ne lésinaient pas sur les moyens. Les films des grands maîtres étaient produits soit par les studios Mosfilm, ce fut le cas de Ptouchko, soit par les studios entièrement affectés à la production des films pour les jeunes - Sojuzdetfilm, créés en 1936, devenus studios Gorki en 1948, dans le cas de Ro'ou. Ils mettaient à disposition de ces réalisateurs des moyens colossaux. Ilya Mouromets (1956), le premier film grand écran en URSS, bat tous les records : 30000 chevaux et 124000 figurants participent au tournage pour chanter la gloire du grand preux du folklore russe. Les ciné-contes figurent également parmi les premiers films en couleur tournés en URSS, comme La Fleur de pierre de Ptouchko (1946) 
qui obtient la même année au festival de Cannes le prix du meilleur film en couleur. Les ciné-contes sont devenus une sorte de laboratoire pour l'expérimentation des effets spéciaux, comme dans La Petite clé d'or où Ptouchko a fait cohabiter les marionnettes et les comédiens en jouant sur les jeux d'échelles. Outre les moyens techniques exceptionnels, les cinéastes étaient entourés de scénaristes exceptionnels comme les dramaturges Nikolaï Erdman (Le Père Frimas, Feu, eau et trompettes de cuivre) ou Evguenij Schwartz ( L'Habile Maria) pour qui le cinéma pour enfants représentait la seule échappatoire.

Avec la disparition des deux pères fondateurs en 1973, la créativité du cinéma pour enfants s'essouffle et l'âge d'or du ciné-conte s'achève. Sur le plan idéologique, on constate un processus de la dépolitisation progressive du conte, appelé à ses débuts à inculquer les valeurs nationalistes, et qui avec le temps se convertit en divertissement pur.

\section{BIBLIOGRAPHIE}

CLARK Katerina (1985), Soviet Novel: History as Ritual, Chicago : Chicago University Press.

KABAKOVA Galina (2005), « Les aventures du conte en Russie », Revue des livres pour enfants, 221, 143-148.

\section{NOTES}

1. Les historiens parlent du rôle central de la chanson dans l'architectonique du cinéma parlant en URSS.

\section{RÉSUMÉS}

L'industrie du cinéma soviétique conçu dès le départ comme un outil de propagande et d'éducation des masses, commence à produire des films pour enfants dès la moitié des années 1920. À l'époque, le conte suscite la méfiance des autorités car à leurs yeux il ne véhicule pas les valeurs de la nouvelle société et le merveilleux est nocif pour la conscience des jeunes bâtisseurs du socialisme; on choisit de leur montrer les aventures de jeunes héros de la guerre civile ou de pionniers qui rendent visite à Lénine. La réhabilitation du conte - et du folklore en général - ne se fera que dix ans plus tard dans le contexte du retour aux valeurs conservatrices. Le folklore sera alors pleinement instrumentalisé dans la promotion du nationalisme soviétique aux accents de plus en plus russes. Les héros des bylines (chants épiques) et des contes envahissent alors les écrans ; à la pointe de la lutte idéologique, ils sont appelés à créer l'image 
du passé glorieux de la Russie et à intimider les ennemis potentiels afin de les dissuader de toute attaque. En même temps, l'industrie du film merveilleux, qui connaît son heure de gloire grâce aux créations d'Alexandre Ptouchko et d'Alexandre Ro'ou, produit également des contes cinématographiques plus divertissants et moins belliqueux. Quels sont les critères de choix des sujets à adapter ? La " préférence nationale » ne s'applique pas forcément aux choix des studios. Ils portent à l'écran des contes déjà populaires grâce aux traductions ou aux adaptations. Dans la filmographie on trouve des contes russes (Morozko, Vassilissa la Belle), mais également des adaptations de contes de Perrault, ou encore des créations plus au moins librement inspirées par le folklore comme La Fleur de pierre d'après le conte de Bajov ou La Fille des neiges (Snégourotchka) d'après la pièce d'Alexandre Ostrovski. Parallèlement, les cinéastes fabriquent des "cross-over ", en mélangeant des personnages et des motifs du folklore russe ou de l'univers d'Andersen. Dans notre présentation, on s'intéressera à la poétique de ces contes cinématographiques ainsi qu'aux défis idéologiques de ces adaptations.

The Soviet film industry designed from the beginning as a tool of propaganda and mass education began to produce children films from the mid 1920'es. At the time, Fairy tales raised the suspicion of the authorities because it did not convey the values of the new society and the wonderful was suspected to be harmful to the conscience of the young builders of socialism. Rehabilitation of the fairy tale -and folklore in general- took place only ten years later in the context of a return to conservative values. Folklore were fully exploited in the promotion of Russian nationalism. The heroes of bylina (epic poem) and tales invaded the screens at the forefront of the ideological struggle, they were called to create the image of the glorious past of Russia and to warn potential enemies. At the same time, the industry of marvelous movie, which knew its heyday thanks to the creations of Alexander Ptushko and Alexander Ro'ou, also producing cinematic tales that are more entertaining and less bellicose. The selection criteria for subjects to adapt "National preference" does not necessarily apply to the choice of the studios. They bring to the screen stories, which are already popular thanks to translations or adaptations. There are some Russian tales (Morozko, Vasilisa the Beautiful), but also adaptations of Perrault's tales as well as more creations freely inspired by folklore as The Stone Flower after the Bajov tale or Snegurochka (the snow Girl) based on the play by Alexander Ostrovsky. Meanwhile, the filmmakers make the "crossover", mixing characters and motifs of Russian folklore or of Andersen's tales. In our presentation, we consider the poetic of the tales films as well as the ideological challenges of these adaptations.

СОВЕТСКАЯ КИНОИНДУСТРИЯ, ПЕРЕД КОТОРОЙ ИЗНАЧАЛЬНО БЫЛИ ПОСТАВЛЕНЫ ЗАДАЧИ ИДЕОЛОГИЧЕСКОЙ ПРОПАГАНДЫ И ВОСПИТАНИЯ МАСС, СТАЛА СИСТЕМАТИЧЕСКИ ПРОИЗВОДИТЬ ФИЛЬМЫ ДЛЯ ДЕТЕЙ С СЕРЕДИНЫ 1920-Х ГГ. НО В ПЕРВЫЕ ГОДЫ СОВЕТСКОЙ ВЛАСТИ СКАЗКИ ВЫЗЫВАЛИ НАСТОРОЖЕННОСТЬ ВЛАСТЕЙ, ПОСКОЛЬКУ В НИХ ОТСУТСТВОВАЛИ НРАВСТВЕННЫЕ ЦЕННОСТИ НОВОГО СТРОЯ, А ИХ ВОЛШЕБСТВО, С ТОЧКИ ЗРЕНИЯ ПСИХОЛОГОВ, МОГЛО ВРЕДНО СКАЗАТЬСЯ НА СОЗНАНИИ БУДУЩИХ СТРОИТЕЛЕЙ СОЦИАЛИЗМА. ПЕРЕСМОТР ОТНОШЕНИЯ К СКАЗКЕ И ФОЛЬКЛОРУ В ЦЕЛОМ ПРОИЗОШЕЛ В СЕРЕДИНЕ 1930-Х ГГ., КОГДА НАСТУПИЛ ВОЗВРАТ К КОНСЕРВАТИВНЫМ ЦЕННОСТЯМ. ФОЛЬКЛОР СТАЛ ШИРОКО ИСПОЛЬЗОВАТЬСЯ В ПРОПАГАНДЕ РУССКОГО НАЦИОНАЛИЗМА, И НА ЭКРАНАХ ВСЕ ЧАЩЕ СТАЛИ ПОЯВЛЯТЬСЯ ГЕРОИ БЫЛИН И СКАЗОК, ПРИЗВАННЫЕ ЗАНЯТЬ ПЕРЕДОВЫЕ ПОЗИЦИИ НА ИДЕОЛОГИЧЕСКОМ ФРОНТЕ. ОНИ ДОЛЖНЫ БЫЛИ ОЛИЦЕТВОРЯТЬ ГЕРОИЧЕСКОЕ ПРОШЛОЕ РУСИ, И В ИХ ЗАДАЧУ ВХОДИЛО ЗАПУГАТЬ ЕЕ ПОТЕНЦИАЛЬНЫХ ВРАГОВ. В ТО ЖЕ ВРЕМЯ БЛАГОДАРЯ АЛЕКСАНДРУ ПТУШКО И АЛЕКСАНДРУ РОУ ПРОИЗВОДСТВО ФИЛЬМОВ ДЛЯ ДЕТЕЙ ПЕРЕЖИВАЕТ НАСТОЯЩИЙ РАСЦВЕТ, И НА ЭКРАНАХ ПОЯВЛЯЮТСЯ БОЛЕЕ РАЗВЛЕКАТЕЛЬНЫЕ И МЕНЕЕ ВОИНСТВЕННЫЕ КИНОСКАЗКИ. КАКОВЫ КРИТЕРИИ ПРИ ВЫБОРЕ СКАЗОК ДЛЯ ЭКРАНИЗАЦИИ? ПРЕДПОЧТЕНИЕ НЕ ВСЕГДА ОТДАЕТСЯ РУССКИМ НАРОДНЫМ СКАЗКАМ, ХОТЯ ИМЕННО ОНИ СОСТАВЛЯЮТ БОЛЬШИНСТВО. 
РЕЖИССЕРЫ ВЫБИРАЮТ САМЫЕ ПОПУЛЯРНЫЕ В ТОМ ЧИСЛЕ БЛАГОДАРЯ ПЕРЕВОДАМ И ЛИТЕРАТУРНЫМ ОБРАБОТКАМ ТЕКСТЫ, ТАК, НАРЯДУ С РУССКИМИ СКАЗКАМИ («МОРОЗКО», « ВАСИЛИСА ПРЕКРАСНАЯ») НА ЭКРАН ПЕРЕНОСЯТСЯ СКАЗКИ ПЕРРО (« ЗОЛУШКА») И ЛИТЕРАТУРНЫЕ ОБРАБОТКИ ФОЛЬКЛОРНЫХ СЮЖЕТОВ («КАМЕННЫЙ ЦВЕТОК» ПО БАЖОВУ, « СНЕГУРОЧКА» ПО ПЬЕСЕ А. ОСТРОВСКОГО). В ТО ЖЕ ВРЕМЯ КИНЕМАТОГРАФИСТЫ СОЗДАЮТ КАРТИНЫ-crossover, В КОТОРЫХ ПЕРЕМЕШАНЫ ГЕРОИ И МОТИВЫ РУССКОГО ФОЛЬКЛОРА ИЛИ ЗАИМСТВОВАННЫЕ ИЗ ЛИТЕРАТУРНЫХ СКАЗОК. В СТАТЬЕ РАССМАТРИВАЮТСЯ ВОПРОСЫ ПОЭТИКИ КИНОСКАЗОК И ПРИНЦИПЫ ИДЕОЛОГИЧЕСКОГО ИСПОЛЬЗОВАНИЯ ФОЛЬКЛОРНОГО МАТЕРИАЛА.

INDEX

Keywords : fairy tale, literary fairy tale, transposition, ideological struggle, poetics, movie motsclesru СКАЗКА, ЛИТЕРАТУРНАЯ СКАЗКА, ТРАНСПОЗИЦИЯ, ИДЕОЛОГИЧЕСКАЯ БОРЬБА, ПОЭТИКА, КИНОСКАЗКА

Mots-clés : conte merveilleux, conte littéraire, transposition, lutte idéologique, poétique, cinéma

\section{AUTEUR}

\section{GALINA KABAKOVA}

Université de Paris 4-Sorbonne 